\title{
Development of a Smart Transformer to Control the Power Exchange of a Microgrid
}

\author{
Wim Willems, Tine L. Vandoorn, Jeroen D. M. De Kooning and Lieven Vandevelde \\ Department of Electrical Energy, Systems \& Automation, \\ Ghent University, Ghent, Belgium, Tine.Vandoorn@UGent.be.
}

\begin{abstract}
A smart transformer enables to control the power exchange between a microgrid and the utility network by controlling the voltage at the microgrid side within certain limits. The distributed generation units in the microgrid are equipped with a voltage-based droop control strategy. This controller reacts on the voltage change, making the smart transformer an element that controls power exchange without the need for communication to other elements in the microgrid. To build a smart transformer, several concepts are possible. In a smart transformer with continuous turns ratio, hereafter referred to as continuous smart transformer, the transformer's microgrid-side voltage can be controlled without voltage steps and the accuracy of the voltage control can be very high. The voltage control of a smart transformer with discrete turns ratio, hereafter referred to as discrete smart transformer, is less accurate, as the output voltage is regulated between several discrete values. In this paper, the development of a continuous and discrete smart transformer will be elaborated. Their validity will be proven by implementing these smart transformers in an experimental test setup. Also, some concepts to improve the control accuracy will be proposed.
\end{abstract}

Index Terms - smart transformer, microgrid, distributed generation units, droop control, on-load tap changing transformer

\section{INTRODUCTION}

[ Microgrids are a future power system configuration providing clear economic and environmental benefits compared to the expansion of the power systems [1]. In a microgrid, producers and consumers exchange energy in a peer-to-peer principle [2]. It is typical that the microgrid is only connected to the macro grid through a single Point of Common Coupling (PCC), so that it can detach itself to work in autonomous island mode operation. When the microgrid works in island mode, the local production must be equal to the local consumption. In case of a shortage of electricity, some consumers will not be able to consume, i.e., load scheduling. Conversely, when too much electricity is generated, some consumers are switched on, or the distributed generation (DG) units can deviate from their maximum power point. When the microgrid is connected to the utility grid, the differences between supply and demand can be eliminated as the utility grid becomes a slack bus. An advantage is that for the rest of the network, the microgrid can be seen as a controllable entity. This provides significant benefits for both the microgrid participants through scaling and

The work of T. Vandoorn is financially supported by a Fellowship of the FWO-Vlaanderen (Research Foundation - Flanders, Belgium). This research has been carried out in the frame of the Interuniversity Attraction Poles Programme initiated by the Belgian Science Policy Office (IAP-VII-02). The research of J. D. M. De Kooning is funded by the Special Research Fund (BOF) of Ghent University (Belgium) aggregating, and for the utility network operator, that does not need to consider all units separately [3].

A requirement for the market participation of a microgrid is that the power exchange between the microgrid and the utility network can be controlled to the reference value which is negotiated in the markets. The microgrid will try to shift its consumption to times of a low market price. It will also shift its consumption to mitigate load peaks. This can be done via a communication network to all decentralised production units and consumers in the microgrid [10]. The power exchange is then controlled by a microgrid central controller that communicates new set points to all grid elements, in such a way that the total consumption/production complies with the reference power exchange, as depicted in Fig. 1. However, this requires a large investment in additional communications, may restrict the systems reliability and can cause delays. Therefore, in [4], another possibility is proposed. Here, the utility network communicates only with the PCC of the microgrid, where the transformer located at the PCC, which is called the smart transformer, alters its microgridside voltage accordingly. The decentralised production units fit their output voltage automatically, depending on the power exchange value that is communicated to the PCC.

Also, integrating large amounts of renewable energy sources in the microgrid requires the ability to control the voltages within certain limits. This can be achieved by load scheduling, but also by implementing upper and lower limits into the smart transformer (ST), or even by implementing a small voltage based-droop into the ST. In this way the ST can work as a controller of power exchange, while also mitigating voltage increases and decreases.

In $\S$ II, an overview of the ST concept is given. In $\S$ III the construction of two STs, i.e. continuous and discrete principles, is presented and explained. In $\S$ IV], some measurements on a laboratory setup are provided in order to clarify the operation of the ST in a microgrid.

\section{OVERVIEW of SMART TRANSFORMER CONCEPT}

In order to reduce the required communication data for controlling the power exchange, the ST concept has been presented in [4]. Instead of exchanging new set points with all microgrid elements, only the ST needs to acquire the set point. As depicted in Fig. 2, the ST is a controlled tap changing transformer that is connected at the point of common coupling (PCC) of the microgrid. The transformer is smart in the sense that its control strategy is able to control the power exchange to a set value by controlling its microgrid-side voltage. 


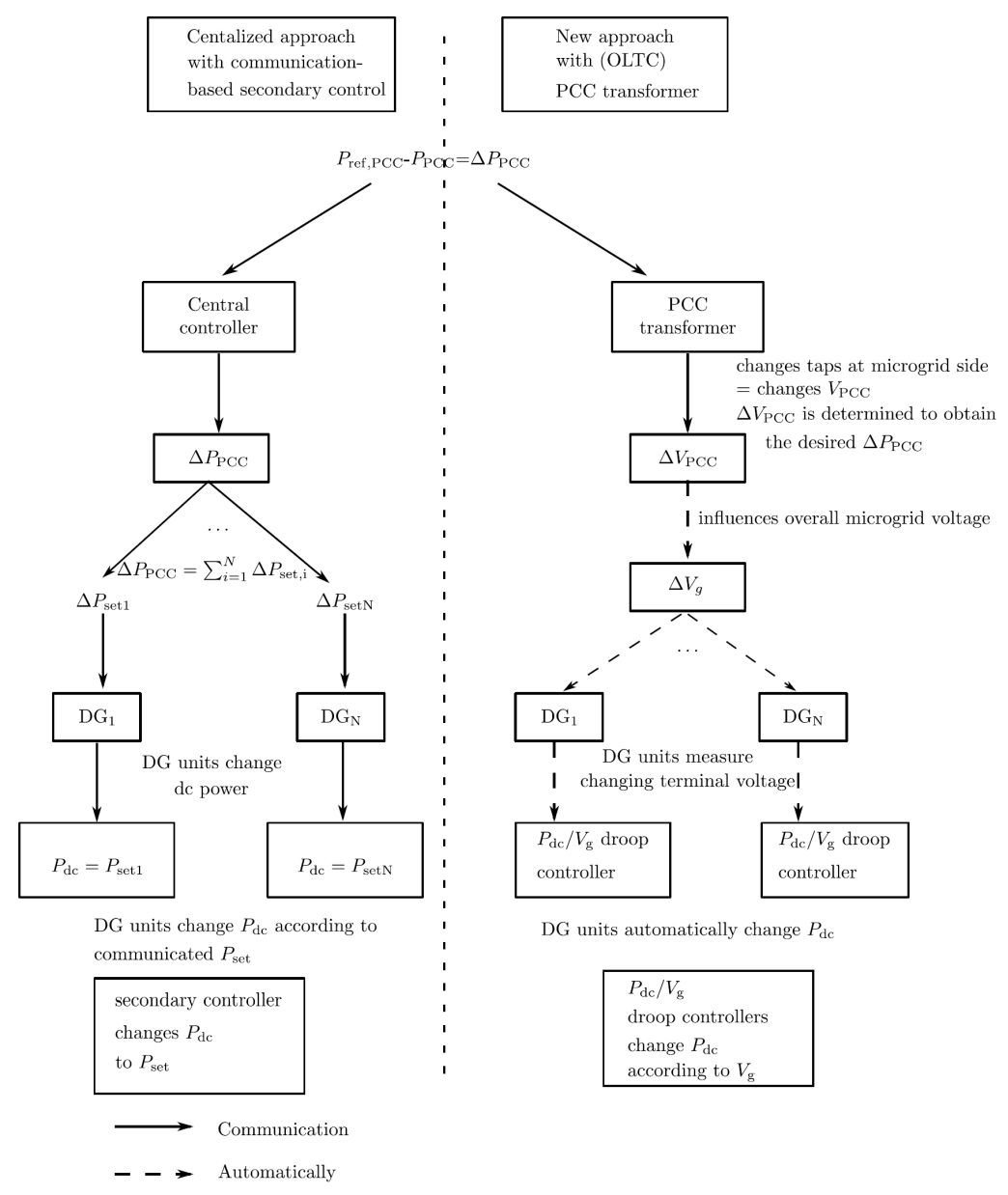

Fig. 1. Smart transformer versus central control to control power exchange between microgrid and utility network [4]

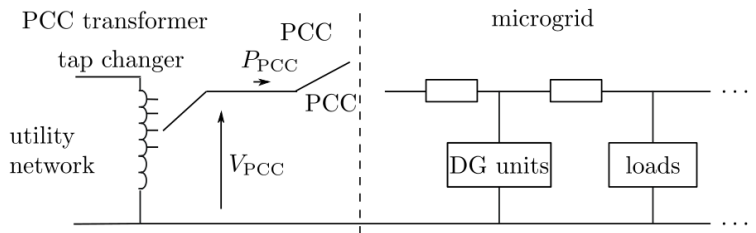

Fig. 2. Smart transformer located at the PCC of a microgrid [4]

In medium-voltage networks, on-load tap changing transformers are sometimes already in place, hence, controlling these as STs requires only little modifications. In the lower voltage networks, most PCC transformers are manual tap changing transformers, from which the voltage can only be controlled offline and not automatically. This puts a significant stress on the electrical grids which face an increased penetration of DG units and significant load increase with the advent of electrical vehicles and heat pumps. Historically, the planning of the low-voltage grids is based on a worst-case scenario ensuring that in case of maximum consumption, the voltage does not drop below the lower voltage limit. Therefore, many tap changing transformers at the beginning of the low- voltage lines are set somewhat above the nominal voltage. However, with the increasing degree of DG units, the risk of overvoltages becomes higher. Also, the planning becomes more difficult because of the larger voltage variations (e.g., sunny day versus night times for photovoltaic panels). Hence, the ability of automatically changing the tap settings becomes more interesting as it is more effective, faster and cheaper to implement than the conventional approach of investing in the grid assets, such as installing more power lines. Therefore, in the future, it is expected to become more beneficial to install an on load tap changer (OLTC). Moreover, it is well-known that a lot of the assets in distribution networks are end-of-life and have to be replaced anyhow in the following years. Hence, the manual transformers can gradually be upgraded to OLTCs.

The OLTC, with smart control strategy, i.e., the ST, controls the PCC power $\left(P_{\mathrm{PCC}}\right)$ by controlling the microgridside voltage $\left(V_{\mathrm{PCC}}\right)$. If the microgrid elements are equipped with the voltage-based droop (VBD) control strategy that is presented in [5], [6], these elements automatically change their input/output power dependent on the grid voltage.

The VBD control strategy, illustrated in Fig. 3, has originally been presented for ensuring a stable operation of lowvoltage islanded microgrids [5]. For the active power control of 


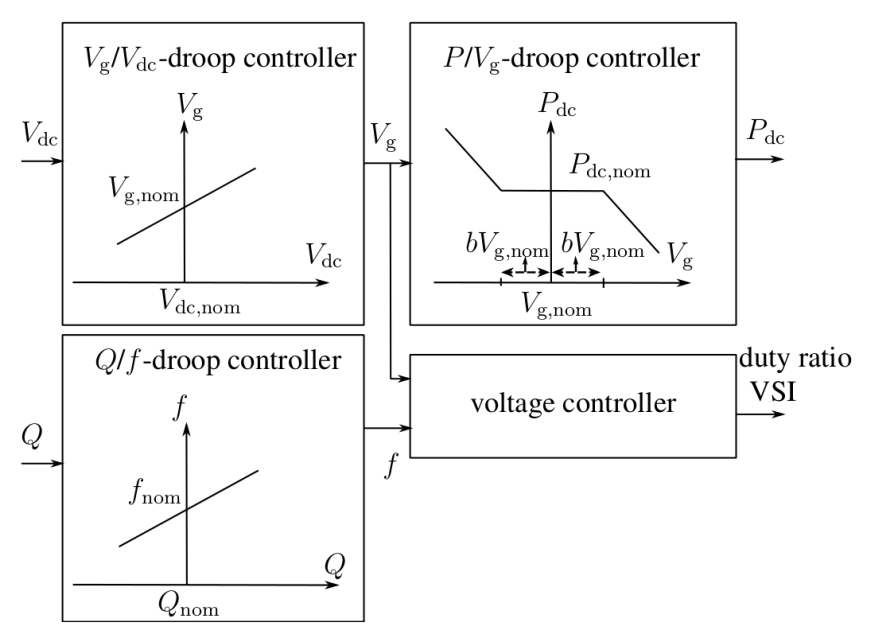

Fig. 3. Voltage-based droop control strategy [4]
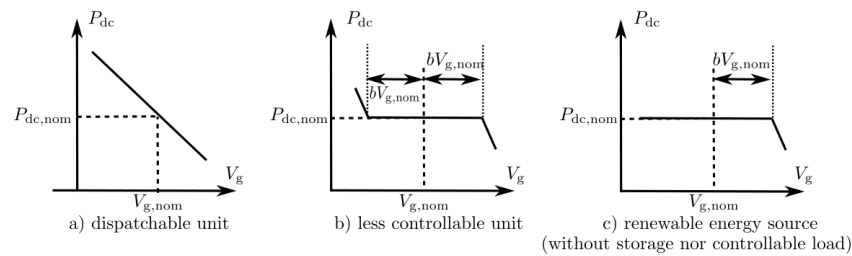

Fig. 4. Constant power bands of dispatchable versus less-dispatchable DG units 4

the DG units, this VBD controller consists of a combination of a $V_{g} / V_{d c}$ droop controller and a $P / V_{g}$ droop controller, with $V_{d c}$ the dc-link voltage, where the power of the dc link is provided by the available renewable energy, and $V_{g}$ the terminal voltage of the DG unit. The former enables power balancing of the DG units ac and dc side and an effective usage of the allowed tolerance on the variations of terminal voltage from its nominal value for grid control. The $P / V_{g}$ droop controller enables to avoid voltage limit violation by changing the input power of the unit. It is combined with constant-power bands that delay the active power changes of the renewables (wide constant-power band) compared to those of the dispatchable DG units (small constant-power band) to more extreme voltages (Fig. 4). For the loads and storage elements, a similar voltage-based control strategy is presented in [6].

As loads, storage elements and DG units in the microgrid react on a changing terminal voltage, the VBD control can be used in a grid-connected microgrid with ST, which is also voltage-controlled to alter the PCC power. For example, when the PCC power injected into the utility network is lower than its pre-agreed value $\left(P_{\mathrm{PCC} \text {,ref }}\right)$, the $\mathrm{ST}$ will lower $V_{\mathrm{PCC}}$. The microgrid DG units react on this voltage drop by increasing their output power, hence, increasing $\left|P_{\mathrm{PCC}}\right|$. Similarly, the active loads can change their consumption based on their terminal voltage [6]. In this way, the PCC power can be controlled by the ST, without the need to communicate new set points to all grid elements as they automatically react. A second advantage is that in this way, a virtual islanded mode is achieved. The utility network is not seen as a slack bus, but is conceived as a constant-power load/generator.

\section{SMART TRANSFORMER REALISATION}

\section{A. General principle}

The power exchange between microgrid and utility network is controlled by a ST. The ST will enable bidirectional power exchange between the utility network and the microgrid, gather information to determine the optimal set point for power exchange and enable to exploit the microgrid as one single unit in the utility network. To control the power exchange between the utility network and the microgrid, the ST has to control the voltage on the microgrid side, working as an OLTC. Hence, the voltage-based control of the microgrid DG units [5] is coupled to a voltage-based control of the ST.

\section{B. Continuous Smart Transformer}

In the continuous ST that was built in the electrical energy laboratory (EELAB) at Ghent university, a variable voltage is obtained by means of a carbon brush which can be rotated on a silver-plated commutator on the circumference of a ring core transformer. For the drive of the brushes, an electric motor is used. The motor is driven by an 'up' or 'down' command. It should be taken into account that the output voltage can be adjusted, both in positive and negative direction. This involves the use of some additional windings on the the side with carbon brush. This configuration provides the ability to regulate the voltage over a very large control range, in theory, the complete control range of the variable transformer.

In practice, however, the transferred power of a transformer is limited due to current limitations in the windings. This causes limitations to the increase of voltage when power is transferred from the side with carbon brush to the side with fixed connection since extremely lowering the brushes can cause a very high current in the side with carbon brush in order to transfer the same power as is desired on the side with fixed connection.

On the variable transformers that are commercially available, there is a limitation on the number of additional windings above the fixed connection point, e.g. to obtain a control range of $0-110 \%$ at the side with carbon brush. As a consequence, it is not possible to obtain a large increase in voltage when power is transferred from the side with fixed connection to the side with carbon brush. Also, it is not possible to obtain a large decrease in voltage when power is transferred from the side with carbon brush to the side with fixed connection.

Another limitation comes from the accuracy of such autotransformers, due to the limited number of turns on a coil. When, e.g., a toroidal transformer is wounded with about 250 turns and the brushes rotate over a non insulated part of a $250 \mathrm{~V}$ winding, the transformer can be controlled with an accuracy of approximately $1 \mathrm{~V}$.

The accuracy of the system can be improved by the use of an additional isolating transformer, which is done in Fig. 5 and Fig. 6. The additional isolating transformer will provide for a voltage increase or decrease. The variable autotransformer is then used to provide a controlled voltage to the primary side of the isolation transformer. The maximum control range is then determined by the winding ratio of the isolation 
transformer. This makes the ST much more accurate for the same accuracy of the brushes drive mechanism. In the variable autotransformer, only a fraction of the total current will flow, namely the current necessary to obtain an increase or decrease in voltage.

One possibility is to provide the variable autotransformer with a fixed tap in the center of the winding, to connect the primary of the isolation transformer with this center tap and to make a connection to the brush on the other terminal of the primary of the isolation transformer, as illustrated in Fig. 5 The secondary of the isolation transformer will then provide a voltage increase or decrease, depending on the setting of the brush on the autotransformer.

Another possibility is the use of a double-pole change-over switch between the two transformers, as illustrated in Fig. 6. Here, the double-pole change-over switch will determine if the isolation transformer will provide for a voltage increase or decrease, depending on the current direction in the additional isolating transformer. The advantage of this possibility is that the accuracy can be two times as high as would be the case with the use of the same transformers in the configuration with center-tap of Fig. 5 .

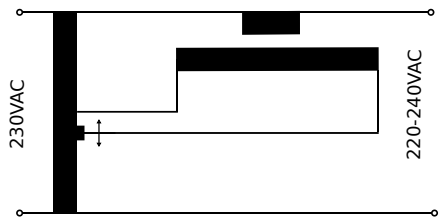

Fig. 5. Continuous smart transformer with second isolation transformer and fixed tap in the center of the autotransformer

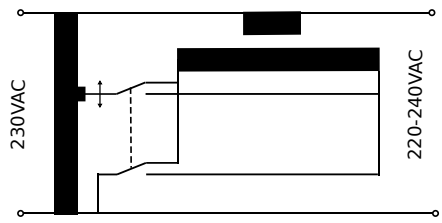

Fig. 6. Continuous smart transformer with second isolation transformer and double-pole change-over switch

\section{Discrete Smart Transformer}

In the discrete ST system that was built in EELAB, one stepdown isolation transformer is used in which the secondary can be made variable by means of a step switch. This principle is similar to the conventional OLTCs and technical details can be found in $[\overline{8}]-[10]$. Switching on the primary would have the advantage that lower currents have to be switched, but this primary winding is often covered by the secondary winding, such that the installation of taps is very difficult. Therefore, in Fig. 7, a configuration is proposed with taps on the secondary side. The direction of the current through the secondary winding is determined by a change-over switch, which can determine whether the isolation transformer will provide for a voltage increase or decrease. The other switches on the secondary winding determine the magnitude of the

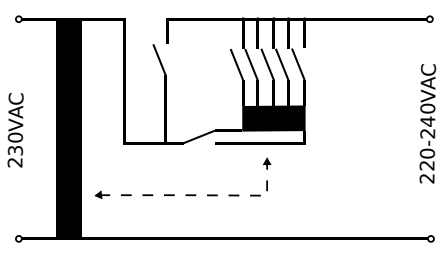

Fig. 7. Discrete smart transformer

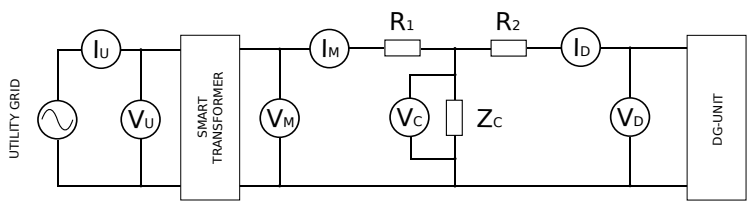

Fig. 8. Laboratory setup

voltage variation. The maximum control range is determined by the winding ratio of the isolation transformer. The power variation is now only step-by-step adjustable, hence the name 'discrete transformer'. Due to the principle of step-by-step adjustments, the discrete ST switching is similar to the switching of conventional OLTCs. However use is made of electronic switches, so switching on the discrete transformer can be preformed much faster (e.g. every cycle) than switching on conventional OLTCs (e.g. every 10 seconds).

\section{MeAsurements}

\section{A. Experimental setup}

For the experiments, a setup with a continuous ST coupled to a fully dispatchable DG-unit was realised. The scheme is shown in Fig. 8, where $V_{M}$ and $I_{M}$ represent the voltage and current measured at the microgrid side of the transformer, while $V_{D}$ and $I_{D}$ represent the voltage and current measured at the DG unit and $V_{C}$ represents the voltage (V) measured at the consumer. From these instantaneous measurements, it is possible to calculate the active powers in the setup, where $P_{M}$ is the active power measured at the microgrid side of the transformer, $P_{D}$ is the active power measured at the DG-unit and $P_{C}$ is the active power measured at the consumer.

\section{B. Measurements with fully dispatchable DG unit}

In the setup, a consumer was added, simulated by a load of $Z_{C}=60 \Omega$. The set-value of the transformer $\left(P_{\mathrm{PCC}, \mathrm{ref}}\right)$ was altered between -200W (2-6s), 200W (8-10s) and 0W (12$14 \mathrm{~s})$, where the minus sign represents power delivered to the microgrid. The measurements of voltages and active power in Fig. 9 show that the ST can follow the set point of $P_{\mathrm{PCC}}$ and that the DG unit automatticaly responds by lowering its output power. This is realised because the voltage at the microgrid side of the transformer becomes higher than the voltage at the DG unit. From the voltage measurements, it is clear that there is a relation between the voltage at the microgrid side of the transformer and the delivered or extracted active power.

\section{Measurements with less dispatchable DG unit}

When a restriction is applied to the delivered power of the DG-unit, it becomes less dispatchable. In the setup of Fig. 8, a power limitation was applied to the DC bus of the 
DG unit to obtain the situation of Fig. $3 \mathrm{c}$, leading to the measurements of Fig. 10 The set value of the transformer $\left(P_{\mathrm{PCC}, \text { ref }}\right)$ was here also altered between $-200 \mathrm{~W}(2-6 \mathrm{~s})$, 200W (8-10s) and 0W (12-14s). The behaviour of the setup is only different in the case of extracting 200W. Here, the total power demanded from the DG unit reaches the maximum power. As a consequence, the voltage at the DG unit drops, resulting to a relatively low voltage in the microgrid. Therefore, it is suggested to implement upper and lower limits to the voltage at the microgrid side of the ST.

\section{CONClusion}

In this paper, two major concepts, the continuous and the discrete ST, are developed and built with focus on the use of the ST as a power controller between utility grid and microgrid. The continuous ST has the advantage that the control of power can be more accurate, where the discrete ST has the advantage that the control of power is fast. The continuous ST concept is validated by including it in an experimental test setup where it is equipped with the necessary measurements and a controller to realise a change of output voltage. It is proven that the power exchange between utility grid and microgrid is being realised by changes in the microgrid-side voltage.
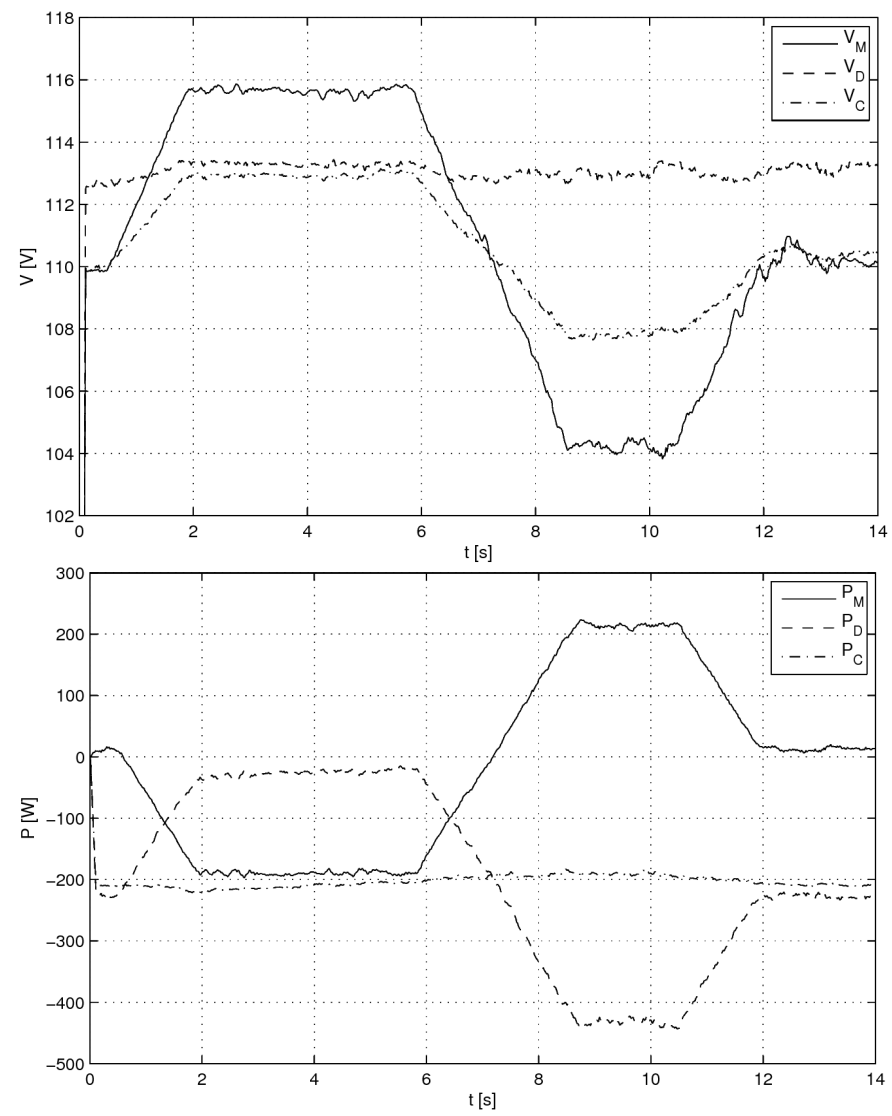

Fig. 9. Measurement on a setup with transformer coupled to a fully dispatchable DG unit.

\section{REFERENCES}

[1] N. Hatziargyriou, H. Asano, R. Iravani and C. Marnay, "Microgrids, An Overview of Ongoing Research, Development, and Demonstration Projects," IEEE Power \& Energy Magazine, pp. 78-94, Jul./Aug. 2007

[2] R. H. Lasseter and P. Paigi, "Microgrid: A Conceptual Solution" Proc. IEEE Power Electron. Spec. Conf. (PESC 2004), Aachen, Germany, 2004

[3] P. M. Costa and M. A. Matos, "Assessing the contribution of microgrids to the reliability of distribution networks," Electrical Power Systems Research, vol. 79, no. 2, pp. 382-389, Feb. 2009

[4] T. L. Vandoorn, J. D. M. De Kooning, B. Meersman, J. Guerrero, and L. Vandevelde, "Voltage-based control of a smart transformer in a microgrid," IEEE Trans. Ind. Electron., vol. 60, no. 4, pp. 1291-1305, Apr. 2013

[5] T. L. Vandoorn, B. Meersman, L. Degroote, B. Renders and L. Vandevelde, "A Control Strategy for Islanded Microgrids with dc-link Voltage Control," IEEE Trans. Pow. Del., vol. 26, no. 2, pp. 703-713, Apr. 2011

[6] T. L. Vandoorn, B. Renders, L. Degroote, B. Meersman, and L. Vandevelde, "Active load control in islanded microgrids based on the grid voltage," IEEE Trans. Smart Grid, vol. 2, no. 1, pp. 139-151, Mar. 2011

[7] A. G. Expsito and D. M. Berjillos, "Solid-State Tap Changers: New Configurations and Applications," IEEE Trans. on Power Delivery, vol. 22, no. 4, pp. 2228-2235, Oct. 2007

[8] J. Faiz and B. Siahkolah, "New Controller for an Electronic Tap Changer Part I: Design Procedure and Simulation Results," IEEE Trans. on Power Delivery, vol. 22, no. 1, pp. 223-229, Jan. 2007

[9] J. Faiz and B. Siahkolah, "New Controller for an Electronic Tap Changer Part II: Measurement Algorithm and Test Results," IEEE Trans. on Power Delivery, vol. 22, no. 1, pp. 230-237, Jan. 2007

[10] J. Guerrero, P. Loh, M. Chandorkar, and T. Lee, "Advanced control architectures for intelligent microgrids - Part I: Decentralized and hierarchical control," IEEE Trans. Ind. Electron., vol. 60, no. 4, pp. 1254-1262, Apr. 2013
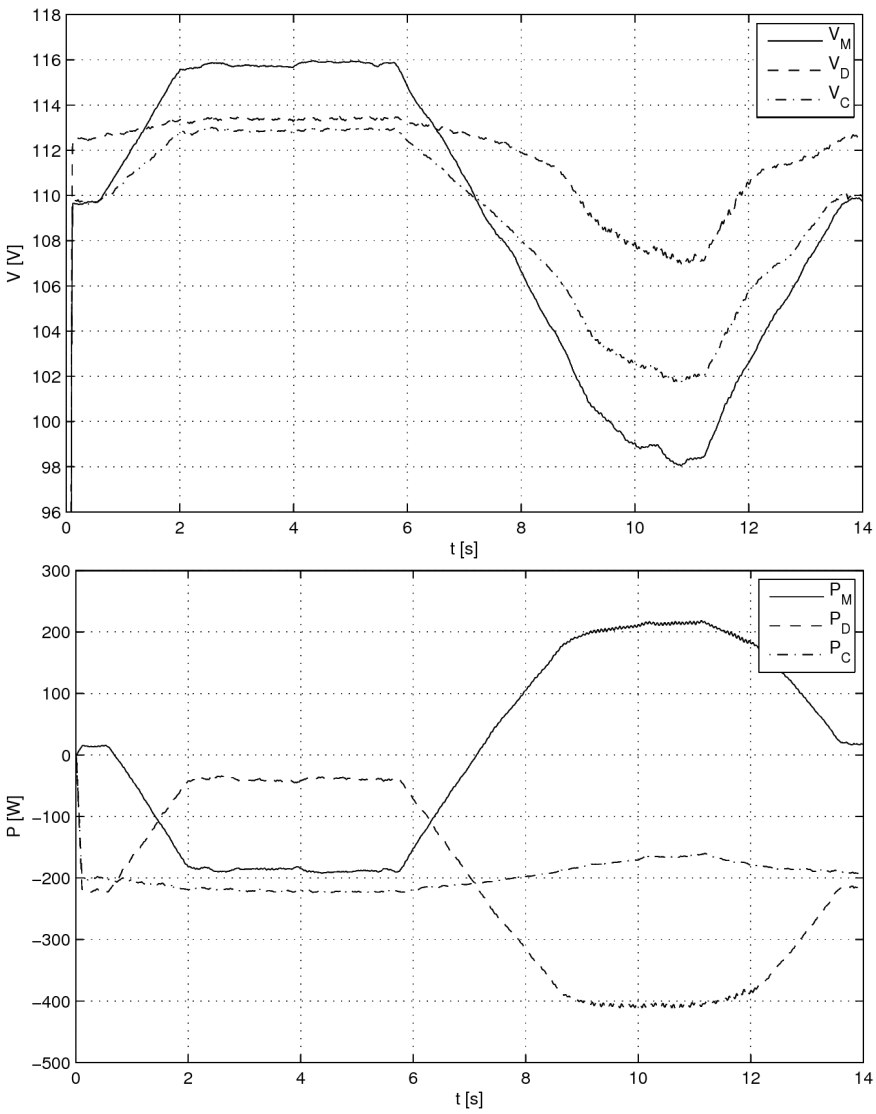

Fig. 10. Measurement on a setup with transformer coupled to a less dispatchable DG unit. 